\title{
Echocardiographic visualization and quantification of mitral complex during mitral repair for severe functional mitral regurgitation
}

\author{
Kikuko Obase, MD, Ichiro Matsumaru, MD, Takashi Miura, MD, and Kiyoyuki Eishi, MD
}

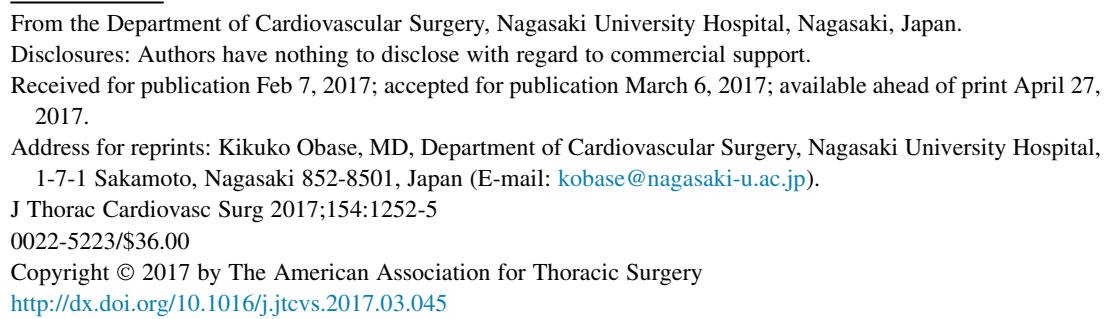

From the Department of Cardiovascular Surgery, Nagasaki University Hospital, Nagasaki, Japan. Disclosures: Authors have nothing to disclose with regard to commercial support.

Received for publication Feb 7, 2017; accepted for publication March 6, 2017; available ahead of print April 27, 2017.

Address for reprints: Kikuko Obase, MD, Department of Cardiovascular Surgery, Nagasaki University Hospital, 1-7-1 Sakamoto, Nagasaki 852-8501, Japan (E-mail: kobase@nagasaki-u.ac.jp).

J Thorac Cardiovasc Surg 2017;154:1252-5 $0022-5223 / \$ 36.00$

Copyright (c) 2017 by The American Association for Thoracic Surgery http://dx.doi.org/10.1016/j.jtcvs.2017.03.045

$\checkmark$ Video clip is available online.

It has been reported that patients with functional mitral regurgitation (MR) who underwent mitral valve repair without recurrent MR had greater left ventricular (LV) reverse remodeling than did the patients who underwent valve replacement. ${ }^{1}$ To prevent recurrent MR completely in severe functional MR, our team has used triple repair, which consists of annuloplasty, anterior leaflet augmentation with a large pericardial patch, and papillary muscle (PM) suspension (Figure 1).

The presented images are from a case of triple repair. Postoperative transesophageal echocardiography shows sufficient leaflet coaptation, which is counted on for prevention of recurrent MR (Figure 2 and Video 1). Threedimensional images of the leaflets in systole reconstructed by a dedicated quantitative software (Real View, Nara, Japan) demonstrate the postoperative improvement of leaflet tethering (Figure 3 and Videos 2 and 3). The preoperative and postoperative distances between mid anterior annulus and each PM tip were 36.4 and $34.9 \mathrm{~mm}$ in anterior PM, and 42.1 and $41.6 \mathrm{~mm}$ in posterior PM. The sutures visualized in Figure 4 (Video 4) are expected for the rence of MR.

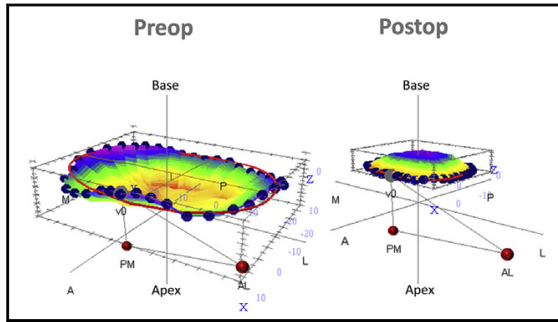

Preoperative and postoperative 3D reconstructed mitral leaflet, annulus, papillary tips.

\section{Central Message}

For successful mitral repair and for further improvement of the quality of surgery, preoperative and postoperative clear visualization and precise quantification of mitral valve complex are essential.

See Editorial Commentaries pages 1256 and 1258.

prevention of further leaflet tethering and thereby recur-

In our experience of severe functional MR cases with LV ejection fraction less than $35 \%$ and LV diastolic dimension greater than $65 \mathrm{~mm}, 11$ patients were treated with mitral annuloplasty alone before 2012; since then, 10 patients have undergone triple repair. Reduction of MR to less than mild at discharge with no operative deaths was achieved in both groups. Cumulative freedoms from recurrent MR at 1 year and 5 years in the mitral annuloplasty series were $90.9 \%$ and $55.7 \%$, respectively, whereas there has been no recurrent MR greater than mild to date in the triple repair group except 1 patient with patch failure.
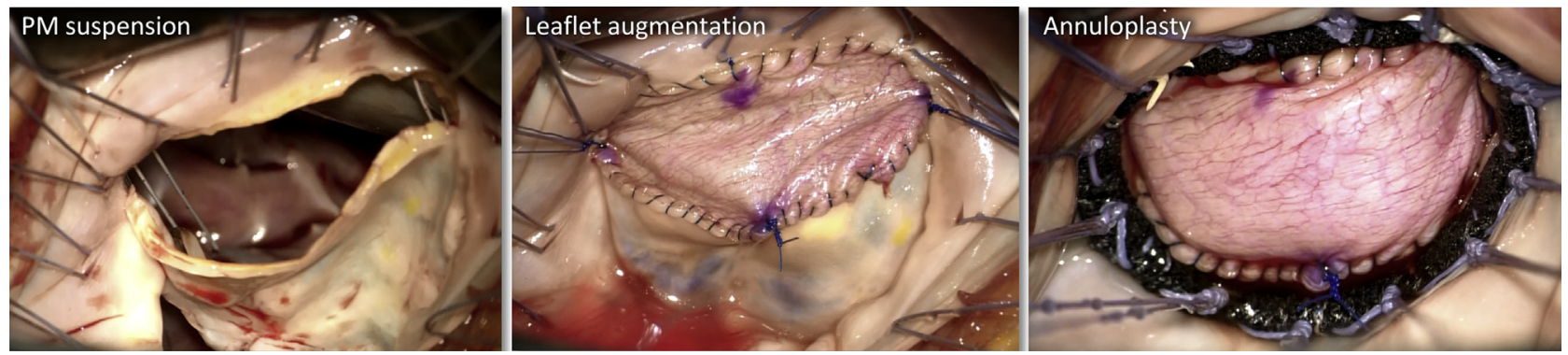

FIGURE 1. The triple repair consisted of papillary muscle $(P M)$ suspension, anterior leaflet augmentation, and annuloplasty. The pericardial patch for the augmentation of the anterior leaflet was designed to cover the whole annular plane. 

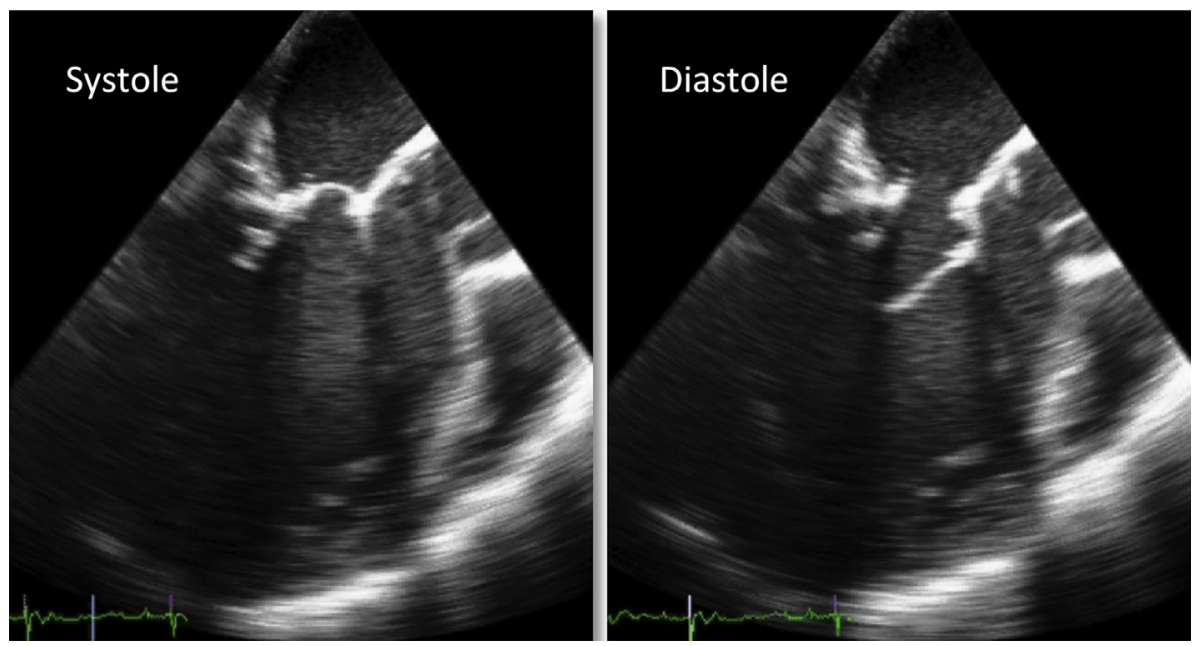

FIGURE 2. Postoperative long-axis view on transesophageal echocardiography visualized deep coaptation and sufficient opening of leaflets. Video of this view is in Video 1, along with postoperative transthoracic echocardiography with and without color Doppler mode.

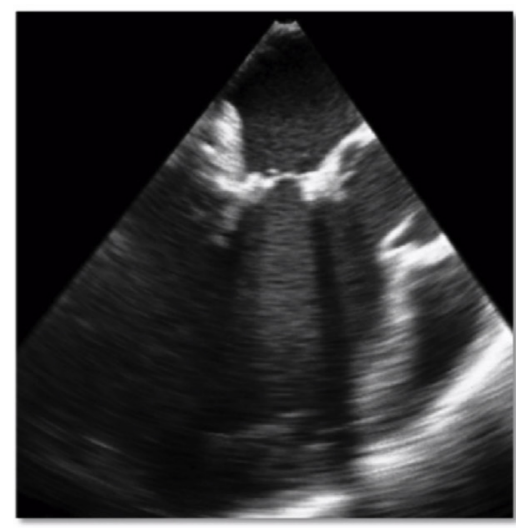

Transesophageal echocardiography

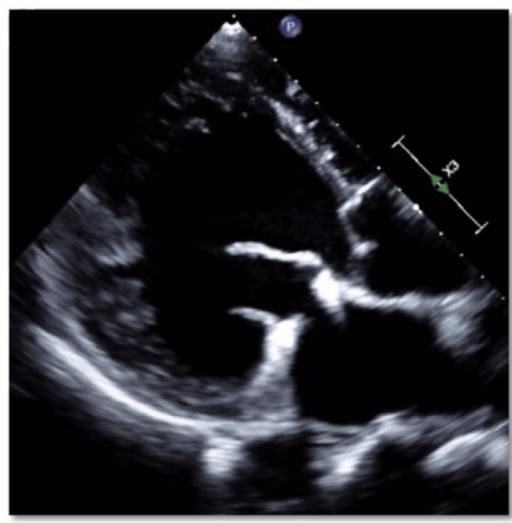

Transthoracic echocardiography

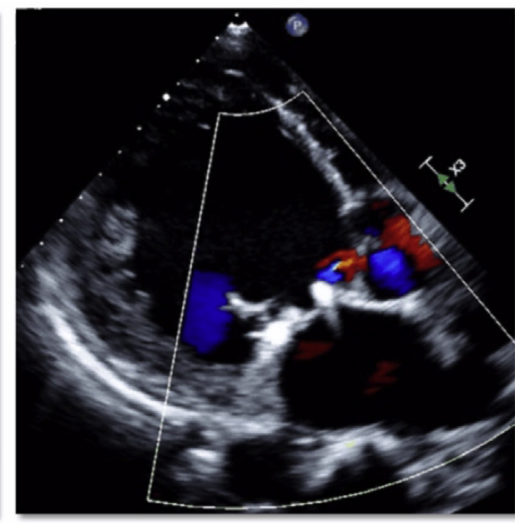

Transthoracic echocardiography Color Doppler mode

VIDEO 1. Postoperative long-axis views by transesophageal echocardiography (left) and by transthoracic echocardiography with (right) and without (middle) color Doppler flow. Video available at: http://www.jtcvsonline.org/article/S0022-5223(17)30555-X/addons.

\section{Preop}

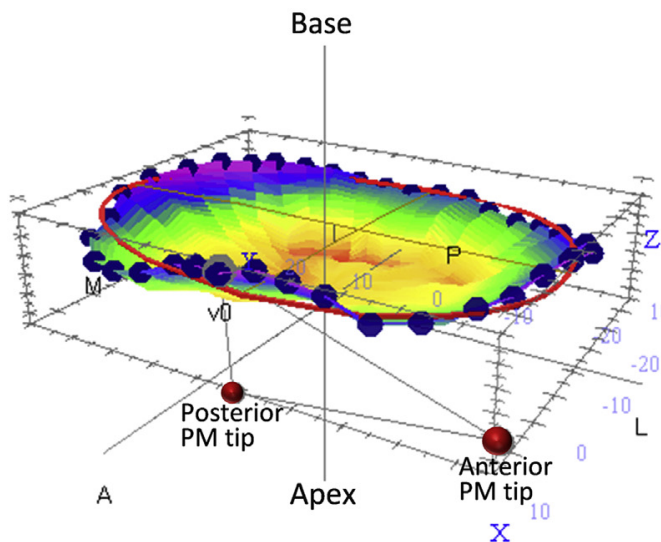

\section{Postop}

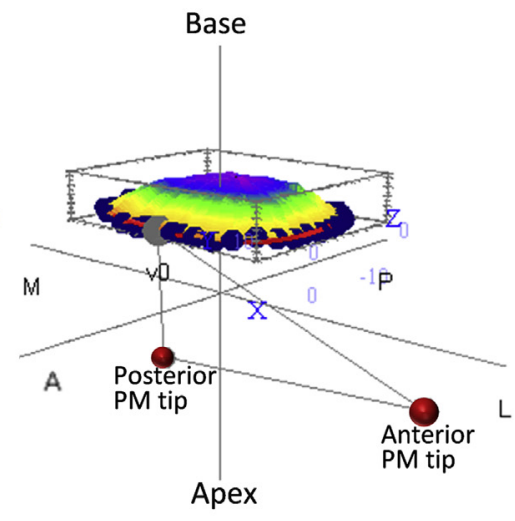

FIGURE 3. Preoperative (left) and postoperative (right) 3-dimensional models of the reconstructed mitral leaflet, the annulus, and the positions of the papillary muscle $(P M)$ tips are shown. Multidirectional observations of these 3-dimensional images are available in Video 2 and Video $3 . M$, Medial; $P$, posterior; $A$, anterior; $L$, lateral. 


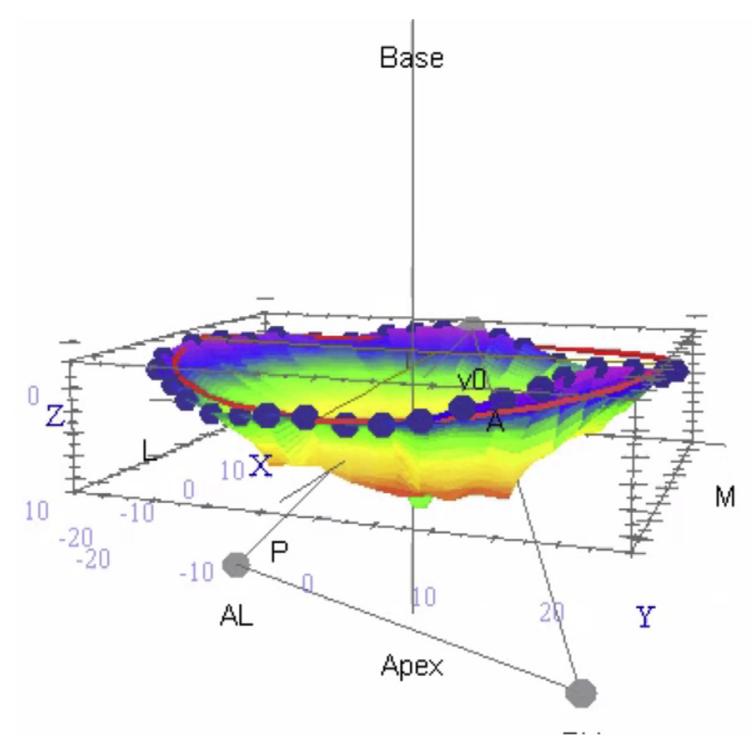

VIDEO 2. Multidirectional observation of 3-dimensional reconstructed preoperative mitral valve complex. $L$, Lateral; $M$, medial; $P$, posterior; $A L$, anterior papillary muscle tip; $P M$, posterior papillary muscle tip. Video available at: http://www.jtcvsonline.org/article/S0022-5223(17)30555-X/addons.

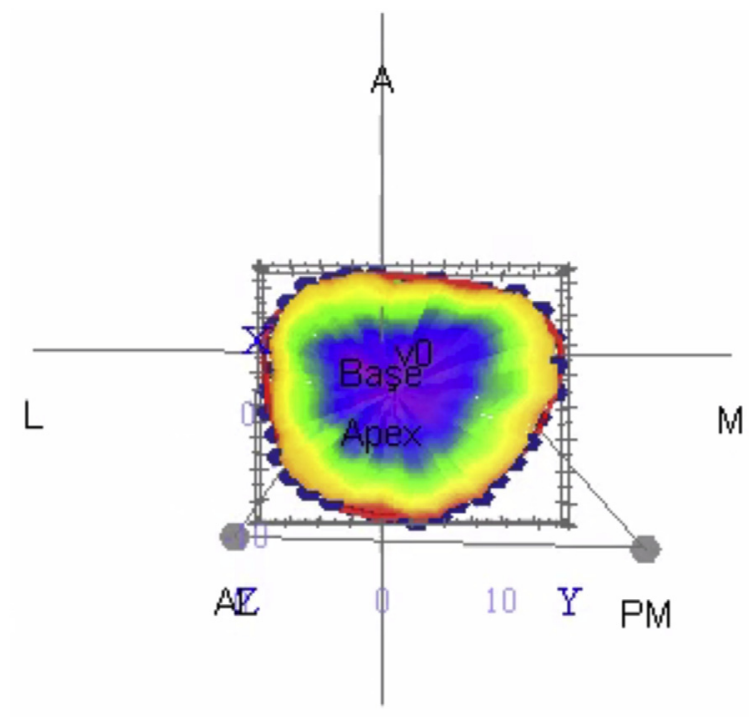

$P$

VIDEO 3. Multidirectional observation of 3D reconstructed postoperative mitral valve complex. $A$, Anterior; $L$, lateral; $M$, medial; $A L$, anterior papillary muscle tip; PM, posterior papillary muscle tip; $P$, posterior. Video available at: http://www.jtcvsonline.org/article/S0022-5223(17)30555-X/addons. 


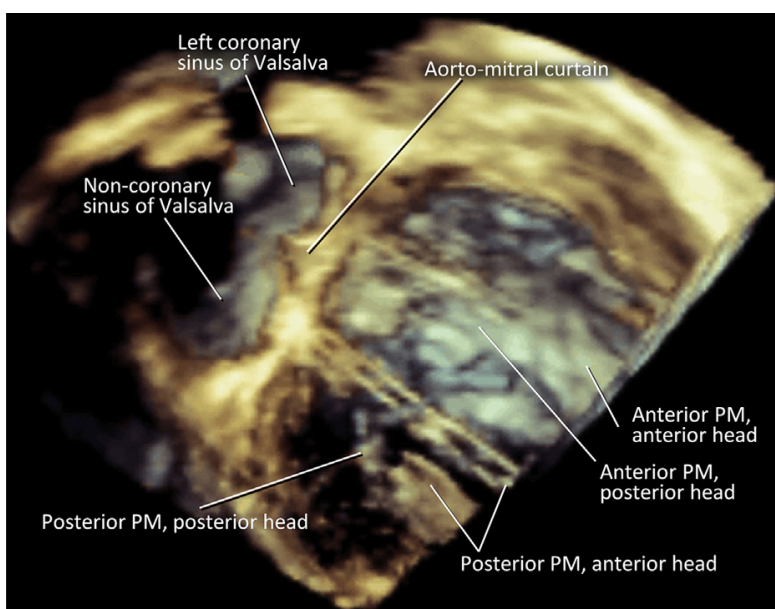

FIGURE 4. Volume-rendering image of transesophageal echocardiography with the transgastric approach allows us to visualize the postoperative submitral apparatus. The pairs of suture for papillary muscle $(P M)$ suspension were clearly visualized (Video 4$)$. The sutures were connected to each fibrous trigone, which can be plainly recognized in this image.
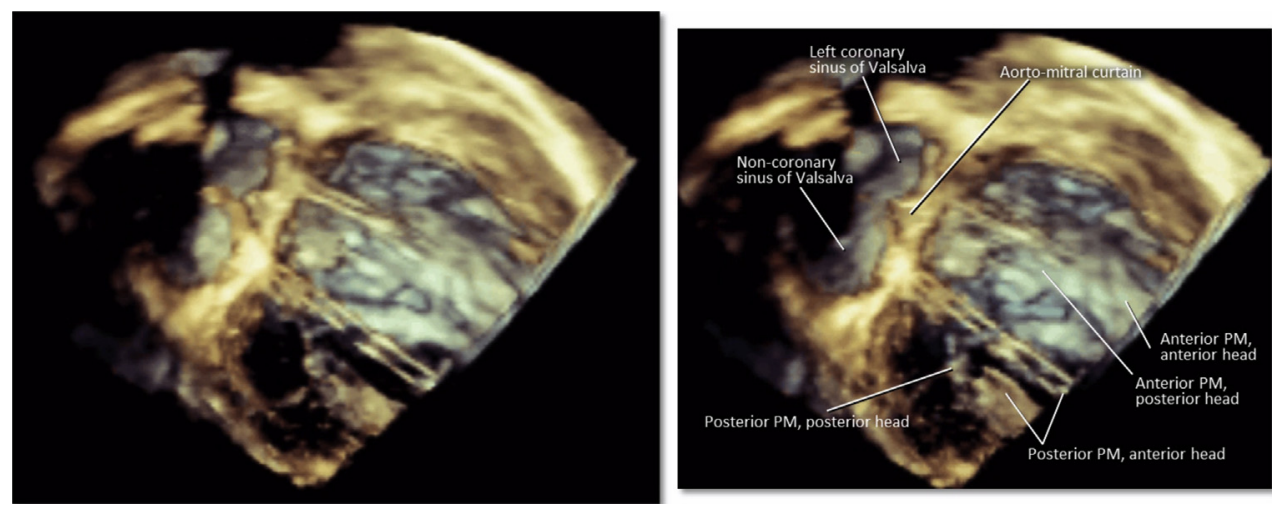

VIDEO 4. Volume-rendering image of postoperative mitral valve complex. $P M$, Papillary muscle. Video available at: http://www.jtcvsonline.org/article/ S0022-5223(17)30555-X/addons.

Further studies in large numbers are warranted to confirm the long-term effectiveness of the procedures. In addition, we believe that optimal imaging and precise quantification in every single case are important for the assessment of the procedures. This will lead to the improvement of the results and therefore outcomes.

\section{Reference}

1. Lorusso R, Gelsomino S, Vizzardi E, D'Aloia A, De Cicco G, Lucà F, et al; ISTIMIR Investigators. Mitral valve repair or replacement for ischemic mitral regurgitation? The Italian Study on the Treatment of Ischemic Mitral Regurgitation (ISTIMIR). J Thorac Cardiovasc Surg. 2013;145:128-39; discussion $137-8$. 